\title{
Desarrollo de las competencias a través del sistema de tareas
}

\section{Development of the competitions through the system of tasks}

\author{
Lucas Palacios* \\ http://dx.doi.org/10.21503/CienciayDesarrollo.2012.v15i2.08
}

\section{RESUMEN}

La teoría científica pedagógica acerca de las competencias ha evolucionando a través de diferentes experiencias educativas y en diferentes partes del mundo contemporáneo; esta indica que la competencia está íntimamente relacionado con la ejecución de un conjunto de tareas, que se trata de una estrategia o un método para resolver los problemas de la vida humana. Esta teoría constituye la hipótesis fundamental que aquí verificamos. La experiencia a la cual se remite nuestra investigación verifica el desarrollo de las competencias en la educación secundaria en una institución educativa de Lima Metropolitana durante el año 2010. Esta experiencia comprueba y/o demuestra que el contenido lógico o la estructura orgánica de las competencias describen una secuencia lógica de tareas, que sirve como estrategia o método en la solución de los problemas; y además indica que el sistema de tareas como estrategia de enseñanza-aprendizaje influye de forma definitiva en el desarrollo de las competencias. Con ello, y a partir de ella, asumimos una nueva conceptualización de las competencias, que da un giro radical respecto de la teoría oficial dominante en la escuela peruana a nivel básico y universitario.

Palabras clave: sistema de tareas y competencias.

\section{ABSTRACT}

"The scientific theory about pedagogy the competencies has evolved through different educational experiences in different parts of the contemporary world, this indicates that the competitions is closely related to the execution of a set of tasks, which is a strategy or method to solve the problems of human life. This theory is the fundamental hypothesis that verify here. The experience, which is referred to our research, check the development of competences in secondary education in an educational institution in metropolitan Lima in 2010. This experience proves and / or shows that the logical content or the organizational structure of the competences described in a logical sequence of tasks, which serves as a strategy or method in solving problems, and indicates that the system tasks such as strategy teaching and learning influence for good in the development the competences. With this and from this we assume a new conceptualization the competences, giving a radical departure from the official theory dominant in Peruvian school and university.

Key words: tacks systems and competitions

\footnotetext{
* Miembro de la Asociación Educativa Vigotsky. E-mail: palaciosvigotsky@hotmail.com
} 


\section{INTRODUCCIÓN}

El presente trabajo aporta evidencias acerca de cómo las competencias describen una secuencia lógica de tareas en cuanto estructura orgánica interna de la competencia. Para ello fue necesario asumir las conclusiones de las investigaciones pedagógicas, reformas educativas y experiencias educativas contemporáneas, en especial el informe de la Comunidad Francesa de Bélgica, el contenido de las evaluaciones PISA y TIMSS, así como las recomendaciones de diferentes organismos internacionales acerca de las competencias. En cuanto todas ellas precisan que las competencias se relacionen directa o indirectamente con los procedimientos, operaciones, secuencia lógica de tareas y actividades que los hombres realizan en la vida diaria, al resolver problemas, en especial en el trabajo productivo, asumimos estas conclusiones como hipótesis central de nuestra investigación: que las competencias son estrategias y/o métodos que describen una secuencia lógica de tareas al resolver los problemas, la misma que describe cómo deben ser aprendidas o desarrolladas. Por ello, para su verificación, aplicamos un sistema de tareas en diferentes grupos de educandos, de diferentes niveles en la formación de las competencias, las mismas que demuestran también cómo el sistema de tareas influye en el desarrollo de las competencias.

Esta investigación planteaba esta hipótesis: la competencia describe una secuencia de tareas y las tareas desarrollan estos procedimientos $\mathrm{u}$ operaciones. El objetivo fue verificar si los participantes a través del sistema de tareas desarrollan y/o aprenden las competencias. Es decir, el objeto de estudio fue el proceso del aprendizaje y/o desarrollo de la competencia por el sistema de tareas. Parte del sistema de tareas o familias de tareas desarrolladas en nuestra experiencia fueron:

Desarrollo del proceso de investigación bibliográfica.
Realización del análisis de textos seleccionados.

Desarrollo de las lecturas de los textos e información de las mismas.

Redacción de ensayos pedagógicos.

Elaboración del plan de tesis.

Desarrollo de las actividades de investigación.

Diseño del plan de carrera.

Diseño de la malla curricular.

Diseño del sílabo.

Procesamiento de la información y redacción de la monografía.

Los instrumentos de investigación en cuanto examen de entrada y salida tuvo el carácter y naturaleza de una metacognición del proceso, en cuanto describe el orden lógico de las tareas correspondientes a la competencia en la realización de las tareas o los problemas planteados en cada asignatura, ya sea en la solución del problema o la elaboración y/o construcción del producto. Se dan las siguientes instrucciones:

La actividad consiste en enumerar o indicar los pasos, etapas, momentos o fases, la secuencia lógica (orden estricto) de las tareas que se han realizado en cualquiera de las situaciones planteadas.

Se sugieren ocho pasos como máximo; sin embargo, no es obligatorio plantear las ocho acciones, por lo que se recomienda formular cinco actividades como mínimo.

\section{EXPERIENCIA Y METODOLOGÍA}

El diseño o proceso de la investigación comprendió:

1. Desarrollo de la experiencia. Se desarrollaron y evaluaron dos competencias por cada asignatura.

El sistema de tareas, como modelo de prueba o la estrategia de enseñan- 
za- aprendizaje para el desarrollo de las competencias, comprende una "familia" de tareas y/o problemas planteados de acuerdo a cada competencia a desarrollar. Se aplicó el sistema de tareas propio de cada asignatura, ejecutadas por los participantes dentro del aula de clase fundamentalmente, en un promedio de nueve (9) talleres (clases o lecciones) de cuatro (4) horas cada uno, en un lapso de un mes, tiempo que duró cada una de las asignaturas indicadas.

\section{Recojo de la información}

- Se tomó una prueba de entrada o de diagnóstico y una prueba de salida para conocer el grado de influencia del sistema de tareas en el desarrollo de la competencia.

\section{Organización de la información}

- De los promedios de cada grupo de la muestra.

$\square$ El proceso de metacognición aplicado permitió que los participantes describieran la secuencia lógica de las tareas correspondientes a cada una de las competencias desarrolladas y evaluadas.

$\square$ Las tareas se ordenaron como la teoría indica.

\section{Análisis de los resultados}

La experiencia permitió verificar los principios del aprender haciendo, de enseñar a aprender y de aprender a aprender.

$\checkmark$ Se comprueba que el desarrollo y/o aprendizaje de las competencias profesionales es una forma de preparación para la vida, de la aplicación de lo aprendido, tanto como método y como evaluación.

\section{Discusión}

- Dada la teoría científica anterior, la investigación demostró a que las competencias profesionales se desarrollan y/o aprenden por y a través de la ejecución de las tareas o la resolución de los problemas aplicados en cada una de las clases o lecciones correspondientes.

En lo fundamental, la presente experiencia sigue los siguientes procedimientos:

\section{Procedimiento 1}

Se aplicó un sistema de tareas correspondiente para desarrollar las competencias de las áreas de historia, geografía y economía de primero y quinto grado de educación secundaria en la I.E. "Nuestra Señora del Carmen" del Cercado de Lima Metropolitana, UGEL 03, durante los dos semestres académicos del año 2010.

De abril a julio se aplicaron las tareas y se tomó una prueba de entrada y de salida a cada tarea y competencia desarrollada en el período indicado.

De agosto a setiembre se aplicaron las tareas y se tomó una prueba de entrada y de salida a cada tarea y competencia desarrollada en el período indicado.

\section{Procedimiento 2}

Para efectos de control del desarrollo de las competencias en otras situaciones distintas de la prueba o experiencia controlada, tomamos una prueba de entrada y de salida para observar los cambios correspondientes, descritos en:

Prueba de entrada en mayo y prueba de salida en noviembre, en las áreas de Matemática, CTA e Historia, Geografía y Economía, en la I.E. "Nuestra Señora de Guadalupe" y I.E. "Argentina" del Cercado de Lima, UGEL 03. Prueba de entrada y de salida en el área de Historia, Geografía y Economía de primer grado en la I.E. "Almirante Miguel Grau”, de Comas, UGEL 04, y en el quinto grado en la 
I.E. "Elvira García y García”, de Pueblo Libre, UGEL 03.

En el caso de las asignaturas de maestría, los exámenes de metacognición comprendieron la enumeración de la secuencia lógica que implicaba cada una de las competencias de la asignatura co- rrespondiente, en función del sistema de tareas aplicadas.

La influencia del sistema de tareas en el desarrollo de las competencias se observa en los gráficos que se presentan a continuación.

En las áreas de Historia, Geografía y Economía:

\section{Promedio de desarrollo por competencias}

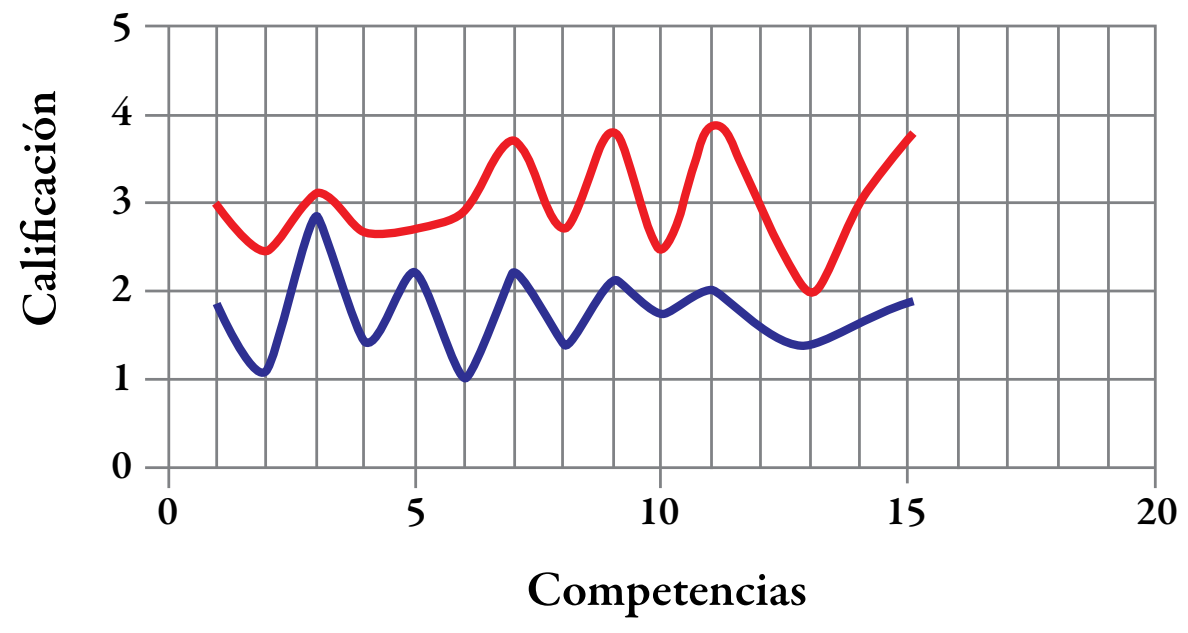

\section{Promedio de desarrollo por asignaturas}

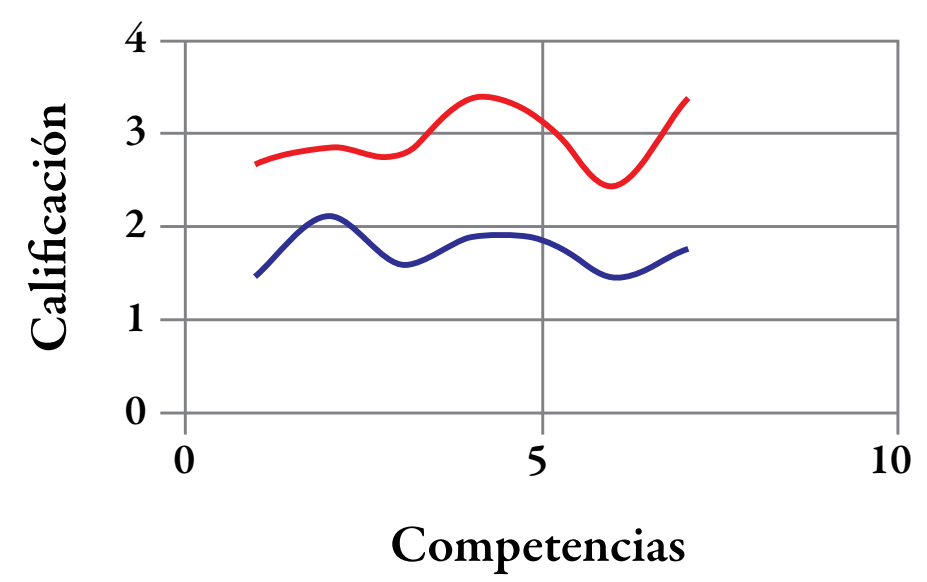




\section{RESULTADOS Y DISCUSIÓN}

Considerando que la pedagogía cubana asume la tarea como célula de la instrucción y como indica la Comunidad Francesa de Bélgica, un sistema de tareas o familia de tareas constituye el medio adecuado para aprender y/o desarrollar las competencias, lo que se halla demostrado en nuestra experiencia como cierta y evidente, por un lado. Por otro, como indica Sidorov ${ }^{1}$, "uno de los grandes físicos de nuestro siglo, Max Laue, formuló en cierta ocasión un aforismo que reza, poco más o menos así: la instrucción es aquello que queda en el hombre cuando todo lo aprendido se olvida. ¿Pero, qué queda si se olvida todo lo que se ha aprendido? Quedan las formas y procedimientos de pensar, los métodos para el examen del objeto que ha de ser estudiado, los procedimientos y formas del pensamiento productivo. El dominio consciente de tales procedimientos, métodos y formas constituye uno de los objetos capitalísimos de la instrucción general". Las investigaciones cubanas acerca de la formación profesional asumen el sistema de tarea, que ratifica el informe de la Comunidad Francesa de Bélgica acerca de las competencias, como lo describe Benjamin Bloom y colaboradores, Sternberg, las evaluaciones PISA, TIMSS, SERCE, o como indica la Comunidad Europea. Es cierto que las competencias describen una secuencialógica de tareas, como hemos observado en el examen tipo metacognición aplicado en las experiencias descritas, el mismo que verifica y se demuestra en los hechos. Presentamos, en tal sentido, la secuencia lógica de tareas de las competencias logradas en nuestra experiencia, en las áreas de Historia, Geografía y Economía. Organiza el proceso histórico de acuerdo al tiempo y espacio social

1. "Leí toda la lectura" o "Busco la información".

2. "Resalto las ideas importantes" o "Subrayo todas las fechas...."

1 Sidorov. Cómo el hombre llegó a pensar.
3. "Saco las fechas y acontecimientos que hay en la hoja".

4. "Escribí los acontecimientos" o "Redacto el acontecimiento".

5. "Analizo bien lo que debo poner...".

6. "Dibujé la línea de tiempo" o "Trazo mi línea”.

7. "Ubiqué los años ordenadamente" u "Ordeno todas las fechas en orden...".

8. "Pongo toda la información en cada fecha" o "Coloco la información ya ordenada".

9. "Si hay dos informaciones (inicio y término del proceso), hago una línea sobre él"

10. "Ordeno y escribo lo que está fechado, así no me confundo" o "Corrijo si está bien".

\section{Reconoce el proceso en la organización de los}

\section{hechos históricos}

1. Organiza bien la escala del tiempo en la línea recta.

2. Ordena y precisa las fechas sobre la línea recta principal.

3. Redacta los acontecimientos registrados en la línea de tiempo.

4. Define el acontecimiento de forma precisa, sin argumentos adicionales.

5. Formula adecuadamente los acontecimientos históricos.

6. Establece segmentos que indican procesos históricos.

7. Traza diferentes segmentos que indican los procesos históricos sobre la línea principio.

\section{Procesa la información en las ciencias sociales}

\section{Registro y recojo de la información}

- "Leo diferentes fuentes".

- “Tomo las ideas principales". "Escojo información más coherente".

- "Recolecto información". "Lo junto y ordeno...".

- "Registro la información”. "Busco imágenes para guiarme". 
- "Plasmo la mejor información de todas las fuentes a que haya recurrido".

\section{Análisis o crítica de la información}

- "Analizo detenidamente cada parte del texto". "Analizo las ideas y la información apropiada”.

- "Comprendo el texto".

- "Comparo diferentes fuentes".

- "Critico todo lo que puedo leer...."

- "Me quedo con la más adecuada y correcta".

\section{Organización y/o síntesis de la información}

- "Organizó las ideas de acuerdo a...."

- "Copio las definiciones, partes, estructura, características, gráficos, cronologías, etc.”.

- "Divido las informaciones en diferentes partes: temas, subtemas, conclusiones".

- "Las organizo en un mapa, tríptico, infografía”.

- "Organizo la presentación o exposición de mi tema”.

Esto demuestra que las competencia se relacionan con las estrategias o los métodos para resolver problemas, en cuanto operaciones y procedimientos de las tareas en la actividad productiva, métodos o estrategias que sirven para resolver problemas (Comunidad Francesa de Bélgica), y verifica que la tarea es la célula de la instrucción (Cuba), en cuanto el sistema de tareas desarrolla las competencias profesionales y las tareas desarrollan las facultades y aptitudes humanas (Cuba). La tarea es la metodología para aprender y/o desarrollar competencias, y describe cierto orden de operaciones y procedimientos (Comunidad Francesa de Bélgica).

\section{CONCLUSIONES}

Dada la hipótesis en cuanto teoría anterior, ya sea como antecedente o bases teóricas que contribuyeron a la investigación y al análisis de la experiencia, se puede concluir que:
1. Resulta cierto que las competencias describen una secuencia lógica de tareas, la misma que forma parte de su estructura orgánica interna.

2. Las competencias constituyen estrategias o métodos para resolver problemas o realizar las tareas.

3. El aprendizaje de las competencias sigue su misma lógica: el sistema de tareas.

4. La evaluación que verifica su aprendizaje y/o desarrollo se basa en las tareas o problemas que generan su desarrollo.

\section{REFERENCIAS BIBLIOGRÁFICAS}

1. Belkys González, Machín; Hernández Fdez, Juana I.; Solano López, Edita; Peraza Vargas, Julio. Sistema de tareas diferenciadas para desarrollar la competencia comunicativa en inglés con fines médicos. Cienfuegos: Facultad de Ciencias Médicas "Dr. Raúl Dorticós Torrado".

2. Denyer, Furnémont, Poulain, Vanloubbeeck (2007). Las competencias en la educación, un balance. México: Fondo de Cultura Económica.

3. Flores Castillo, Francis Edie (2008). Las competencias que los profesores de educación básica movilizan en su desempeño profesional docente. Tesis Doctoral. Universidad Complutense de Madrid.

4. Iglesias León, Míriam; Cañedo Iglesias, Carlos; Corona Martínez, Luis; Cortés Cortés, Manuel (2010). "Sistema de tareas docentes: su contribución al modelo de formación de los profesionales cubanos". Cuadernos de Educación y Desarrollo. Vol. 2, No 13. Eumed-Net de la Universidad de Málaga.

5. OCDE Organización para la Cooperación y el Desarrollo Económico (2006). Marco de la evaluación. Conocimientos y habilidades en ciencias, matemáticas y lectura. Programa para la Evaluación Internacional de Alumnos. PISA, Santillana Educación S.L. para la edición española. 
6. Parlamento Europeo (2006). "Recomendación del Parlamento Europeo y del Consejo, de 18 de diciembre de 2006, sobre las competencias clave para el aprendizaje permanente". Diario Oficial L 394 de 30.12.2006.

7. Rodríguez Cobián, Armando Antonio (2009). Sistema de tareas docentes para el desarrollo de habilidades en estudiantes de medicina, en el contexto del policlinico universitario. Tesis para optar al grado científico de máster en educación. Cienfuegos: Cuba.

8. Sidorov, M. (1991). Cómo el hombre llegó a pensar. Lima: Editorial Perú Andino.

9. Sternberg, Robert; GRIGORENKO, Elena (2010). "Enseñanza de la inteligencia exitosa para alumnos superdotados y talentos". Versión de Ferrando, Mercedes; Hernández, Daniel; Ferrándiz, Carmen y Bermejo, Rosario. REIFOP, 13 (1). Revista Electrónica Interuniversitaria de Formación del Profesorado, 13 (1), 111-118, 2010.

10. TIMSS 2007 (2009). Guia del usuario para la base de datos internacional. Preguntas de ciencias y matemática. Cuarto Curso de Educación Primaria. IEA
11. Valdés Rojas, Martha Beatriz (2008). Sistema de tareas docentes con enfoque interdisciplinario para la formación laboral de los alumnos en la secundaria básica. Tesis presentada en opción al grado científico de Doctor en Ciencias Pedagógicas. Instituto Superior Pedagógico Félix Varela Morales. Cuba: Editorial Universitaria del Ministerio de Educación Superior de la República de Cuba.

12. Varios autores (2010). "Sistema de tareas docentes: su contribución al modelo de formación de los profesionales cubanos". Cuadernos de Educación y Desarrollo. Vol. 2, No 13.

13. Varios autores (2008). Experiencia de aplicación del sistema de tareas en la Nueva Universidad Cubana. Cienfuegos: Proyecto Ramal de Investigación.

14. Varios autores (2005). "Sistema de tareas diferenciadas para desarrollar la competencia comunicativa en inglés con fines médicos". Revista Electrónica de las Ciencias Médicas. Cienfuegos: Medisur. 